\title{
Quantum toy model for black-hole backreaction
}

\author{
Clovis Maia ${ }^{1,2}$ and Ralf Schützhold ${ }^{1, *}$ \\ ${ }^{1}$ Institut für Theoretische Physik, Technische Universität Dresden, 01062 Dresden, Germany \\ ${ }^{2}$ Instituto de Física Teórica, Universidade Estadual Paulista, 01405-900, São Paulo, SP, Brazil
}

(Received 25 July 2007; published 9 November 2007)

\begin{abstract}
We propose a simple quantum field theoretical toy model for black-hole evaporation and study the backreaction of Hawking radiation onto the classical background. It turns out that the horizon is also "pushed back" in this situation (i.e., the interior region shrinks) though this backreaction is not caused by energy conservation but by momentum balance. The effective heat capacity and induced entropy variation can have both signs - depending on the parameters of the model.
\end{abstract}

DOI: 10.1103/PhysRevD.76.101502

PACS numbers: 04.62.+v, 04.70.Dy

\section{INTRODUCTION}

Black holes are arguably the most simple and at the same time most intriguing objects in the Universe. The no-hair theorem states that they can fully be described by a small set of parameters such as their mass $M$ and angular momentum $J$. Yet our standard picture of black holes contains many striking properties: Even though black holes should be completely black classically, they emit Hawking radiation due to quantum effects [1]. This evaporation process causes the black hole (horizon) to shrink in the absence of infalling matter due to the backreaction of Hawking radiation. Therefore, black holes possess a negative heat capacity [2], i.e., the temperature grows with decreasing energy. Extrapolating this picture till the final stages of the evaporation, the black hole should end up in an explosion, where its temperature blows up and thus effects of quantum gravity should become important. Perhaps most fascinating is the observation that the second law of thermodynamics apparently [3] requires to assign an entropy $S$ to the black hole, which is determined by the horizon surface area $A$ via $S=A / 4$ (in natural units with $\hbar=G=c=1$ ).

Taking the analogy between black holes and thermodynamics seriously provides a very consistent picture, which has been confirmed by various gedanken experiments [3,4] considering the construction of heat engines with black holes, etc. It almost seems as if nature was trying to give us some hints regarding the underlying structure which unifies quantum theory and gravity - which we do not fully understand yet. In order to comprehend these hints better, it might be useful to ask the question of whether (and how) the aforementioned properties depend on the detailed structure of the Einstein equations or whether they are more universal. For example, the study of condensedmatter based black-hole analogues [5,6] shows that Hawking radiation is a fairly robust quantum phenomenon [7], which just requires the occurrence of an effective horizon and thus is quite independent of the Einstein

*schuetz@theory.phy.tu-dresden.de equations. In contrast, the introduction of a black-hole entropy with the desired properties seems to rely on the Einstein equations.

In the following, we try to further disentangle universal features from properties which are specific to black holes (e.g., Einstein equations, rotational symmetry, conserved Arnowitt-Deser-Misner (ADM) mass). To this end, we propose a toy model which captures some of the relevant features of black holes and allows us to study the backreaction of the emitted Hawking radiation onto the classical background solution.

\section{TOY MODEL}

In the toy model we are going to discuss, the gravitational field will be represented by a real scalar field $\psi$ in $1+1$ dimensions with the Lagrangian $(\hbar=1)$

$$
\mathcal{L}_{\psi}=\frac{1}{2}\left(\dot{\psi}^{2}-c_{\psi}^{2}\left[\partial_{x} \psi\right]^{2}\right)-V(\psi) .
$$

With respect to the propagation speed $c_{\psi}$ of the $\psi$ field, this form is Lorentz invariant. The potential $V(\psi)$ is supposed to be very stiff, i.e., the field $\psi$ is assumed to be heavy in the sense that it can be approximated by a classical field. For definiteness, we choose the sine-Gordon potential $V(\psi) \propto 1-\cos \left(\psi / \psi_{0}\right)$, but other potentials admitting stable localized solutions would also work. The global ground state $\psi=0$ then corresponds to a vanishing gravitational field whereas a kink (topological defect) models a black (or white) hole horizon

$$
\psi(x)=-4 \psi_{0} \arctan \left(\exp \left\{-\xi\left[x-x_{\text {kink }}\right]\right\}\right) .
$$

The position $x=x_{\text {kink }}$ of the kink at rest is arbitrary and its width $1 / \xi$ is determined by $V(\psi)$ and $c_{\psi}$. In comparison to other models of black holes (see, e.g., [8,9]), the advantage of the above setup lies in the topologically protected stability and localization of the kink (see also [10]). This topological defect behaves very similar to a particle, i.e., the position of the kink is a dynamical variable with a well-defined inertial mass $M_{\text {eff }}$ and the associated kinetic energy $E_{\text {kin }}=M_{\text {eff }} c_{\psi}^{2}\left(\left[1-\dot{x}_{\text {kink }}^{2} / c_{\psi}^{2}\right]^{1 / 2}-1\right)$. 
In order to study Hawking radiation and its impact on the kink, we consider a massless quantum field $\phi$ coupled to the heavy field $\psi$ via the coupling constant $g$

$$
\mathcal{L}_{\phi}=\frac{1}{2}\left(\left[\partial_{t} \phi+g \psi \partial_{x} \phi\right]^{2}-c_{\phi}^{2}\left[\partial_{x} \phi\right]^{2}\right) .
$$

Note that the velocity $c_{\phi}$ of the light (massless) field may differ from $c_{\psi}$. The propagation of the light field $\phi$ in the approximately classical background $\psi$ is completely analogous to that in a gravitational field described by the Painlevé-Gullstrand-Lemaître metric (cf. [5,6])

$$
d s^{2}=\left(c_{\phi}^{2}-v^{2}\right) d t^{2}-2 v d t d x-d x^{2},
$$

where $v=g \psi$ denotes the local velocity of freely falling frames. A horizon occurs if this velocity $v$ exceeds the speed of light $c_{\phi}$. Based on the analogy to gravity, we may also derive the pseudo energy-momentum tensor of the $\phi$ field with respect to the above metric $g^{\mu \nu}$

$$
T_{\mu \nu}=\frac{2}{\sqrt{-g}} \frac{\delta \mathcal{A}_{\phi}}{\delta g^{\mu \nu}}=\left(\partial_{\mu} \phi\right)\left(\partial_{\nu} \phi\right)-\frac{1}{2} g_{\mu \nu}\left(\partial_{\rho} \phi\right)\left(\partial^{\rho} \phi\right) .
$$

The associated energy density $T_{0}^{0}$ of the light field

$$
\mathcal{H}_{\phi}=\frac{1}{2}\left(\left[\partial_{t} \phi\right]^{2}+\left(c_{\phi}^{2}-v^{2}\right)\left[\partial_{x} \phi\right]^{2}\right)
$$

contains negative parts beyond the horizon $v^{2}>c_{\phi}^{2}$. Of course, this is precisely the reason why effects like Hawking radiation are possible.

However, an energy density which is not bounded from below seems unphysical and typically indicates instabilities (already on the classical level). In order to avoid this problem, we may add an extra term which does not modify the linearized low-energy behavior of our model

$$
\mathcal{L}_{\phi}^{\mathrm{reg}}=\mathcal{L}_{\phi}-\alpha^{2}\left(c_{\phi}^{2}-v^{2}\right)^{2}\left[\partial_{x} \phi\right]^{4}-\frac{1}{16 \alpha^{2}},
$$

but generates a positive definite energy density

$$
\mathcal{H}_{\phi}^{\mathrm{reg}}=\frac{1}{2}\left(\left[\partial_{t} \phi\right]^{2}+\left[\alpha\left(c_{\phi}^{2}-v^{2}\right)\left[\partial_{x} \phi\right]^{2}+\frac{1}{4 \alpha}\right]^{2}\right) .
$$

In the exterior region $c_{\phi}^{2}>v^{2}$, the classical ground state is still given by $\phi=0$, but beyond the horizon $c_{\phi}^{2}<v^{2}$, we have $2 \alpha\left(\partial_{x} \phi\right)=\left(v^{2}-c_{\phi}^{2}\right)^{-1 / 2}$. Thus, the classical ground state profile would not be differentiable at the horizon, i.e., the term $\left[\partial_{x} \phi\right]^{2}$ in the energy density, for example, would be ill-defined. This problem can be cured by adding another term (which again does not modify the low-energy behavior) and we finally arrive at the total Lagrangian of our toy model

$$
\mathcal{L}_{\text {full }}=\mathcal{L}_{\psi}+\mathcal{L}_{\phi}^{\text {reg }}-\beta^{2}\left[\partial_{x}^{2} \phi\right]^{2} .
$$

The last term smoothens the classical ground state profile at the horizon and induces a superluminal dispersion relation $(\Omega+v k)^{2}=c_{\phi}^{2} k^{2}+2 \beta^{2} k^{4}$ at large wave numbers.
Assuming that the effective surface gravity $\kappa$ in Eq. (15) is much smaller than the knee frequency where the dispersion relation changes $\kappa \ll c_{\phi} / \beta$, such a superluminal dispersion relation does not lead to alterations of the Hawking effect. On the contrary, it helps us to solve the trans-Planckian puzzle and to understand the origin of Hawking radiation in this model: Since wave packets with large $k \gg c_{\phi}^{2} / \beta$ have a superluminal group velocity $d \omega / d k \gg c_{\phi}$, they may overcome the frame-dragging speed $v$ and thus approach the horizon from the inside. During that process, the inhomogeneity of $v$ (i.e., $v$ is smaller on the front end of the wave packet than on its rear end) stretches the wave packet and reduces its wave number (analogous to the gravitational redshift) and thereby its group velocity. Eventually, the wave packet gets "ripped apart" and one part (the Hawking radiation) escapes into the exterior region whereas the remaining part (the infalling partner particle) is swept away into the interior domain. Assuming that the wave packet was in its ground state initially (for large $k$ ), the combined quantum state of the outgoing Hawking quanta and their infalling partner particles is a pure state, but the reduced density matrix of the Hawking radiation alone is thermal with the Hawking temperature [7]. Having established this thermal emission for our model, we may now ask what are its consequences.

\section{BACKREACTION}

The equation of motion of the light field can be derived from the Lagrangian above

$$
\left(\partial_{t}+v \partial_{x}\right)\left(\partial_{t}+\partial_{x} v\right) \phi=c_{\phi}^{2} \partial_{x}^{2} \phi+\mathcal{O}\left(\partial_{x}^{4}\right),
$$

where $\mathcal{O}\left(\partial_{x}^{4}\right)$ denote the higher-order $\alpha$ and $\beta$ terms we added for stability and regularity reasons. Similarly, the heavy field evolves according to

$$
\ddot{\psi}-c_{\psi}^{2} \partial_{x}^{2} \psi=V^{\prime}(\psi)-g\left[\partial_{t} \phi+g \psi \partial_{x} \phi\right] \partial_{x} \phi+\mathcal{O}\left(\partial_{x}^{4}\right) .
$$

From the full set of equations, we see that the kink profile in Eq. (2) together with $\phi=0$ exactly solves the classical equations of motion (though it is not the ground state). However, the impact of quantum fluctuations changes this picture: For $2 \pi g \psi_{0}>c_{\phi}$, the kink acts as a black-hole horizon and thus emits Hawking radiation. Of course, the energy/momentum given off must come from somewhere and hence this quantum effect should have some impact on the classical kink background.

In order to estimate the quantum backreaction, we quantize the fields $\phi \rightarrow \hat{\phi}$ as well as $\psi \rightarrow \hat{\psi}$ and employ a mean-field expansion $\hat{\psi}=\psi_{\mathrm{cl}}+\delta \hat{\psi}$ where $\psi_{\mathrm{cl}}$ denotes the classical kink profile in Eq. (2) and $\delta \hat{\psi}$ as well as $\hat{\phi}$ are supposed to be small (i.e., $\hat{\phi}, \delta \hat{\psi} \ll \psi_{\mathrm{cl}}$ ). Taking the expectation value of Eq. (11) and comparing it with Eq. (5), we find that the lowest-order contributions of the 
quantum backreaction force are just given by the expectation value of the pseudo energy-momentum tensor [11]

$$
\left[\partial_{t}^{2}-c_{\psi}^{2} \partial_{x}^{2}-V^{\prime \prime}\left(\psi_{\mathrm{cl}}\right)\right]\langle\delta \hat{\psi}\rangle \approx-g\left\langle\hat{T}_{1}^{0}\right\rangle .
$$

Remembering the covariant energy-momentum balance

$$
\nabla_{\mu} T_{\nu}^{\mu}=\frac{1}{\sqrt{-g}} \partial_{\mu}\left(\sqrt{-g} T_{\nu}^{\mu}\right)-\frac{1}{2} T^{\alpha \beta} \partial_{\nu} g_{\alpha \beta}=0,
$$

we find that $T_{1}^{0}$ denotes the momentum density $\pi_{\phi} \phi^{\prime}$, which varies with position in general. In contrast, the energy flux $T_{0}^{1}$ measured with respect to the stationary frame is constant $\partial_{x}\left\langle\hat{T}_{0}^{1}\right\rangle=0$ for a kink at rest.

Fortunately, the expectation value $\left\langle\hat{T}_{\nu}^{\mu}\right\rangle$ can be calculated analytically for a scalar field in $1+1$ dimensions. In the Unruh state (which is the appropriate state for describing black-hole evaporation), one obtains [12]

$$
\left\langle\hat{T}_{1}^{0}\right\rangle=\frac{4 v c_{\phi}\left(\kappa^{2}-\left[v^{\prime}\right]^{2}-\gamma v v^{\prime \prime}\right)-\kappa^{2}\left(c_{\phi}+v\right)^{2}}{48 \pi c_{\phi}^{3} \gamma^{2}},
$$

with $\gamma=1-v^{2} / c_{\phi}^{2}$ and the effective surface gravity $\kappa$ determining the Hawking temperature

$$
T_{\text {Hawking }}=\frac{\kappa}{2 \pi}=\frac{1}{2 \pi}\left(\frac{d v}{d x}\right)_{v^{2}=c_{\phi}^{2}} .
$$

Note that $\left\langle\hat{T}_{1}^{0}\right\rangle$ calculated in the Unruh state is regular across the black-hole horizon $v=-c_{\phi}$, but singular at the white-hole horizon $v=+c_{\phi}$. [The Israel-HartleHawking state is regular at both horizons, cf. Eq. (18).] Far away from the kink/horizon $v \rightarrow 0$, we just get the usual thermal flux $\left\langle\hat{T}_{1}^{0}\right\rangle=-\kappa^{2} /\left(48 \pi c_{\phi}\right)$.

The corrections induced by the quantum backreaction can be visualized by incorporating them into an effective potential $V_{\text {eff }}$ via

$$
V_{\mathrm{eff}}^{\prime}(\psi)=V^{\prime}\left(\psi_{\mathrm{cl}}\right)-g\left\langle\hat{T}_{1}^{0}\right\rangle .
$$

For the classical potential $V(\psi)$, all minima $\psi \in 2 \pi \psi_{0} \mathbb{Z}$ occur at the same energy $V=0$. However, the effective potential $V_{\text {eff }}$ is distorted such that the central minimum is lower than the next one describing the black-hole interior $V_{\text {eff }}(\psi=0)<V_{\text {eff }}\left(-2 \pi \psi_{0}\right)$. In this sense, the exterior region is effectively energetically favorable and thus the horizon starts to move inwards, i.e., the black hole shrinks. Alternatively, the same result can be derived directly from Eq. (12) via classical time-dependent perturbation theory around the kink solution. The differential operator on the left-hand side of Eq. (12) possesses a continuum of gapped propagating (delocalized) modes with $\omega^{2}>0$ and one localized zero mode $\propto 1 / \cosh \left(\xi\left[x-x_{\text {kink }}\right]\right)$ with $\omega=0$, which just corresponds to a translation of the kink position [13]. After expanding the source term $-g\left\langle\hat{T}_{1}^{0}\right\rangle$ in Eq. (12) into these modes, the perturbations in the continuous spectrum $\omega^{2}>0$ just propagate away from the kink-whereas the spatial overlap between $-g\left\langle\hat{T}_{1}^{0}\right\rangle$ and the zero mode determines the acceleration $\ddot{x}_{\text {kink }}<0$ of the kink position.

\section{ENERGY AND MOMENTUM}

In contrast to the fluid analogues for black holes (with a steady inflow and outflow of energy and momentum), for example, the kink considered here represents a welllocalized object, which allows us to ask the question of where the force pushing back the horizon comes from. In most cases, such an analysis is rather complicated [14] because the contribution of the $\phi$ field to the total energymomentum tensor $\mathcal{T}^{\mu \nu}$ (defined with respect to the Minkowski metric $\partial_{\mu} \mathcal{T}^{\mu \nu}=0$ ) differs from the pseudo energy-momentum tensor $\nabla_{\mu} T^{\mu \nu}=0$ in (5) defined with respect to the effective metric (4). Fortunately, these difficulties are absent in our toy model since the mixed components of both tensors coincide $\mathcal{T}_{\nu}^{\mu}=T_{\nu}^{\mu}$. The energy density $T_{0}^{0}$ is given by Eq. (6) and the classical expression for the momentum flux density just reads $T_{1}^{1}=-T_{0}^{0}$ due to conformal invariance of the scalar field in $1+1$ dimensions. Note, however, that the quantum expectation values differ due to the trace anomaly [12]. The energy flux density

$$
T_{0}^{1}=\dot{\phi} \frac{\partial \mathcal{L}}{\partial \phi^{\prime}}=\dot{\phi}\left[v \dot{\phi}+\left(v^{2}-c_{\phi}^{2}\right) \phi^{\prime}\right],
$$

also deviates from the momentum density $T_{1}^{0}$ in Eqs. (11) and (12) for $v \neq 0$.

Far away from the kink, we may estimate the above quantities by employing the geometric-optics approximation and replacing $\dot{\phi} \rightarrow \Omega$ and $\phi^{\prime} \rightarrow k$. For solutions of the dispersion relation $(\Omega+v k)^{2}=c_{\phi}^{2} k^{2}+\mathcal{O}\left(k^{4}\right)$ corresponding to the outgoing Hawking radiation and its infalling partner particles, the energy density per normalized amplitude $T_{0}^{0}=c \Omega^{2} /(c-|v|)$ changes its sign at the horizon, cf. Eq. (6). The energy flux density $T_{0}^{1}=c \Omega^{2}$ is constant and positive everywhere (which is even true beyond the geometric-optics approximation). Note that $\Omega$ is conserved as we are considering a quasistationary scenario. Thus, the total energy budget is balanced since the outgoing Hawking radiation carries away positive energy, but the infalling partners have a negative energy.

The momentum density $T_{1}^{0}=-c \Omega^{2} /(c-|v|)^{2}$, on the other hand, turns out to be negative everywhere - or more precisely, far away from the kink, cf. the exact expression (14) with $\Omega \sim \kappa$. Thus the momentum flux density $T_{1}^{1}=$ $-c \Omega^{2} /(c-|v|)$, i.e., the pressure, also changes sign at the horizon. (The trace anomaly vanishes in the asymptotic region $v^{\prime}=v^{\prime \prime}=0$ far away from the kink where the geometric-optics approximation applies $T_{1}^{1}=-T_{0}^{0}$.) Consequently, while the Hawking particles carry away positive momentum and push back the kink, their infalling partner particles act in the opposite way and pull on the kink. In summary, the momentum is not balanced and thus 
the kink starts to move, i.e., the black-hole interior region shrinks.

Of course, the above considerations can only be applied in the weak-field limit, i.e., when the acceleration $\ddot{x}_{\text {kink }}$ of the kink is negligibly small during the typical emission time $\mathcal{O}(1 / \kappa)$ for one Hawking quantum $\left|\ddot{x}_{\text {kink }}\right| \ll \kappa c_{\phi}$. Using the above momentum balance $M_{\text {eff }} \ddot{x}_{\text {kink }} \propto \kappa^{2}$, this translates to $M_{\text {eff }} \gg \kappa / c_{\phi}^{2}$, which is analogous to the usual semiclassical requirement $M_{\text {Planck }} \gg \kappa / c^{2}$ in the blackhole case and will be automatically satisfied for sufficiently stiff potentials $V(\psi)$ in Eq. (1) leading to an approximately classical background solution $\psi_{\mathrm{cl}}$ in Eq. (2).

\section{THERMODYNAMICS}

The application of thermodynamic concepts to our toy model (in analogy to real black holes) presents some difficulties and ambiguities: Considering the heat capacity $C=d E / d T$, for example, we would associate $T$ with the Hawking temperature (15). The variation of the internal energy $d E$, however, could be identified with the heat given off by the Hawking radiation $d E=\delta Q \propto \kappa^{2} d t$ during a time interval $d t$ or, alternatively, with the change of the kinetic energy of the kink $E=M_{\text {eff }} \dot{x}_{\text {kink }}^{2} / 2$ (for $\dot{x}_{\text {kink }}^{2} \ll$ $\left.c_{\psi}^{2}\right)$. Since the kink does not possess a conserved ADM mass, these quantities will be different in general. Either way, the heat capacity $C=d E / d T$ could be positive as well as negative (or even infinite - at the turning point where $d T=0$ ) since the Hawking temperature can be increased $d T>0$ or decreased $d T<0$ by the quantum backreaction of the evaporation process [15].

Similar ambiguities apply to the entropy $d S=d E / T$. Choosing $d E=\delta Q \propto \kappa^{2} d t$ just reproduces the entropy flow of the Hawking radiation into the exterior regionwhich is of course indeed thermal. Inserting the kinetic energy $E=M_{\text {eff }} \dot{x}_{\text {kink }}^{2} / 2$, on the other hand, does also yield a total differential $d S=d E\left(\dot{x}_{\text {kink }}\right) / T\left(\dot{x}_{\text {kink }}\right)$ and hence allows us to define an alternative entropy concept. However, using this definition, we could violate the 2 nd law since the kink can be accelerated or slowed down by incident coherent radiation (carrying zero entropy).

\section{CONCLUSIONS}

Modeling the black hole (horizon) by a stable topological defect in the form of a kink, we were able to derive the quantum backreaction of the resulting evaporation process. It turns out that the kink/horizon is also pushed inwards as in a real black hole though, in contrast to the gravitational case, this backreaction force is not caused by energy conservation but by momentum balance. Energetically, the expansion of the horizon should be favorable because the minimum energy density in exterior region $\phi=\psi=0$ lies far above $1 /(4 \alpha)^{2}>0$ the ground state in the interior region. Hence, going beyond the linear analysis performed here, one might suspect that the $\phi$ field approaches its ground state via nonlinear (quantum) instabilities until the evaporation stops.

Note that in the Israel-Hartle-Hawking state with

$$
\left\langle\hat{T}_{1}^{0}\right\rangle_{\mathrm{IHH}}=\frac{4 v c_{\phi}\left(\kappa^{2}-\left[v^{\prime}\right]^{2}-\gamma v v^{\prime \prime}\right)}{48 \pi c_{\phi}^{3} \gamma^{2}},
$$

the horizon is still pushed inwards-i.e., this state does also not correspond to the thermal equilibrium for the combined system [kink in Eq. (2) plus $\phi$ field]. This observation is related to the fact that the Lagrangian of our model in Eqs. (1) and (3) is only invariant under time reversal if we simultaneously invert $\psi \rightarrow-\psi$, which does not leave the background kink solution unaffected-but turns it into an antikink (corresponding to a white-hole horizon $v \rightarrow-v$ ).

Further thermodynamical concepts such as heat capacity or entropy (variation) cannot be defined unambiguously and can have both signs - depending on the considered parameters [16]. Thus, in contrast to Hawking radiation, which is quite robust (i.e., independent of the Einstein equations) and just requires the existence of an effective horizon, the heat capacity and the entropy concept strongly depend on the underlying structure (e.g., Einstein equations). This observation may cast some doubt on the proposed explanation of black-hole entropy solely based on the entanglement entropy of the Hawking radiation (the latter is universal, the former not).

Together with the results in [9], our calculations and the energy-momentum considerations above suggest that the resulting backreaction force "pushing" the horizon inwards may also be universal. Speculating a bit further, one may ask whether the observation that the horizon "wants" to shrink could be somehow linked to the blackhole information paradox because a shrinking horizon (in contrast to a growing one) might perhaps entail the possibility of finally releasing the information which fell into the black hole.

\section{ACKNOWLEDGMENTS}

R.S. acknowledges valuable discussions with Ted Jacobson, Bill Unruh, Renaud Parentani, and others at the workshop From Quantum to Emergent Gravity: Theory and Phenomenology (SISSA, Trieste, Italy 2007) and Gerard Dunne, Daniel Grumiller, and others at the 7th workshop on Quantum Field Theory under the Influence of External Conditions (University of Leipzig, Germany, 2007) as well as support by the Emmy-Noether Programme of the German Research Foundation (DFG, No. SCHU 1557/1-2,3). C. M. is indebted to G. Matsas for the support, the ITP at TU-Dresden for the hospitality, and Fundação de Amparo à Pesquisa do Estado de São Paulo for financial support. 
[1] S. W. Hawking, Nature (London) 248, 30 (1974); Commun. Math. Phys. 43, 199 (1975).

[2] For simplicity, we are considering black holes without charge $Q=0$ and angular momentum $J=0$. Otherwise we would have to distinguish different heat capacities in analogy to $c_{p}$ and $c_{V}$ in thermodynamics, as can be seen by comparing the first law of thermodynamics $d E=$ $T d S-p d V$ with the first law of black-hole dynamics $d M=\kappa d A /(8 \pi)+\Omega_{H} d J+\Phi d Q$, where $\Omega_{H}$ is the angular velocity (at the horizon) and $\Phi$ is the (electrostatic) potential.

[3] J. M. Bardeen, B. Carter, and S. W. Hawking, Commun. Math. Phys. 31, 161 (1973); J. D. Bekenstein, Lett. Nuovo Cimento Soc. Ital. Fis. 4, 737 (1972); Phys. Rev. D 7, 2333 (1973); 9, 3292 (1974); 12, 3077 (1975).

[4] See, e.g., W. G. Unruh and R. M. Wald, Phys. Rev. D 25, 942 (1982).

[5] W. G. Unruh, Phys. Rev. Lett. 46, 1351 (1981).

[6] C. Barceló, S. Liberati, and M. Visser, Living Rev. Relativity 8, 12 (2005), http://www.livingreviews.org/lrr2005-12; and references therein.

[7] See, e.g., W. G. Unruh and R. Schützhold, Phys. Rev. D 71, 024028 (2005); T. Jacobson and D. Mattingly, Phys. Rev. D 61, 024017 (1999); S. Corley, Phys. Rev. D 57, 6280 (1998); R. Brout, S. Massar, R. Parentani, and P. Spindel, Phys. Rev. D 52, 4559 (1995); T. Jacobson, Phys. Rev. D 44, 1731 (1991).

[8] See, e.g., O. B. Zaslavskii, Classical Quantum Gravity 20, 2963 (2003); S. Nojiri and S. D. Odintsov, Phys. Rev. D 59, 044003 (1999); and references therein.

[9] R. Balbinot, S. Fagnocchi, A. Fabbri, and G. P. Procopio, Phys. Rev. Lett. 94, 161302 (2005); R. Balbinot, S. Fagnocchi, and A. Fabbri, Phys. Rev. D 71, 064019 (2005).

[10] T. Jacobson and T. Koike, in Artificial Black Holes, edited by M. Novello, M. Visser, and G. Volovik (World Scientific, Singapore, 2002).

[11] In a similar way, one could calculate the backreaction of the quantum fluctuations of the $\psi$ field itself. However, symmetry arguments show that these corrections do not generate any force on the kink.

[12] P. C. Davies, S. A. Fulling, and W. G. Unruh, Phys. Rev. D 13, 2720 (1976).

[13] A.R. Bishop, J.A. Krumhansl, and S.E. Trullinger, Physica D (Amsterdam) 1, 1 (1980); M. B. Fogel, S. E. Trullinger, A. R. Bishop, and J. A. Krumhansl, Phys. Rev. B 15, 1578 (1977); J. Rubinstein, J. Math. Phys. (N.Y.) 11, 258 (1970).

[14] M. Stone, Phys. Rev. E 62, 1341 (2000); Phys. Rev. B 61, 11780 (2000); Artificial Black Holes, edited by
M. Novello, M. Visser, and G. Volovik (World Scientific, Singapore, 2002).

[15] There are several different effects: Because of the distortion of the effective potential $V_{\text {eff }}$, the shape of the kink deviates from the classical profile (2). This deviation is governed by the aforementioned continuum modes $\omega^{2}>$ 0 . Furthermore, the kink starts to move-which is described by the zero mode. The motion of the kink, in turn, implies a Doppler shift of the Hawking radiation. Finally, even in the rest frame of the kink, the position of the horizon $x_{h}$ changes since the kink velocity $\dot{x}_{\text {kink }}$ effectively reduces the local frame-dragging speed $v$ and therefore the surface gravity $\kappa=v^{\prime}\left(x_{h}\right)$ may change. As a result of all these effects, the heat capacity depends on many parameters $\left(c_{\phi}, c_{\psi}\right.$, and $g \psi_{0}$, etc. $)$ and may assume negative as well as positive values. In order to demonstrate this sign ambiguity, let us consider the case $c_{\psi} \gg c_{\phi}$ for simplicity. In this limit, the continuum modes $\omega^{2}>0$ are very fast and hence the change of the shape of the kink can be neglected, i.e., the quantum backreaction induces a rigid motion of the kink only. As another simplification, the transformation of the $\psi$-field into the rest frame of the kink is just a Galilei transformation due to $c_{\psi} \gg c_{\phi}$. The new horizon position is then simply determined by $v\left(x_{h}\right)=-c_{\phi}+\dot{x}_{\text {kink }}$. Linearizing this equality together with $\kappa=v^{\prime}\left(x_{h}\right)$, we find that the variation $\delta \kappa$ of the surface gravity induced by the acceleration of the kink $\delta \dot{x}_{\text {kink }}$ is determined by $\delta \kappa=v^{\prime \prime}\left(x_{h}\right) \delta \dot{x}_{\text {kink }} / \kappa$. Since $v^{\prime \prime}\left(x_{h}\right)$ can be positive or negative (depending on the relation between $c_{\phi}$ and $g \psi_{0}$ ), the temperature measured in the rest frame of the kink could change in both directions. The temperature in the laboratory frame acquires an additional Doppler shift, which is given by $\delta \kappa=$ $-\kappa \delta \dot{x}_{\text {kink }} / c_{\phi}$. The relative strength of the two competing effects (Doppler shift and horizon displacement) is given by $c_{\phi} v^{\prime \prime} /\left(v^{\prime}\right)^{2}$, which can be above or below one. Ergo, both temperatures (in the kink frame and in the laboratory frame) may increase or decrease due to the backreaction of Hawking radiation, i.e., the heat capacity can be positive or negative (or even infinite — at the turning point where $\delta T=0)$.

[16] The fate of the evaporating kink at late times also depends strongly on the parameters. E.g., for $c_{\psi} \ll c_{\phi}$, the kink will behave like a massive relativistic particle under the influence of an accelerating force. I.e., it will be accelerated towards $\left|\dot{x}_{\text {kink }}\right| \uparrow c_{\psi}$ while its slope becomes constantly steeper due to the Lorentz contraction. As a result, the surface gravity grows - accelerating the kink even more. This mechanism will result in a blowup where eventually the higher-order terms will become important. 